\title{
The benefits of etoposide capsules as maintenance therapy for patients with extensive-stage small cell lung cancer: a prospective two-stage, two-center study
}

\author{
Cuicui Zhang ${ }^{1 \#}$, Jianchun Duan ${ }^{2 \#}$, Zhen $\mathrm{He}^{1}$, Li Yang ${ }^{1}$, Sen Yang ${ }^{1}$, Zhe Zhang ${ }^{1}$, Yang Liu ${ }^{3}$, Rui Wan ${ }^{2}$, \\ Lin Lin $^{2}$, Xuan Wu ${ }^{1}$, Wei Wang ${ }^{4}$, Qiming Wang ${ }^{1}$, Jie Wang ${ }^{2}$
}

${ }^{1}$ Department of Internal Medicine, Affiliated Cancer Hospital of Zhengzhou University, Henan Cancer Hospital, Zhengzhou, China; ${ }^{2}$ State Key Laboratory of Molecular Oncology, Department of Medical Oncology, National Cancer Center and Cancer Hospital, Chinese Academy of Medical Sciences and Peking Union Medical College, Beijing, China; ${ }^{3}$ Department of Radiotherapy, Affiliated Cancer Hospital of Zhengzhou University, Henan Cancer Hospital, Zhengzhou, China; ${ }^{4}$ Henan Medical Association, Zhengzhou, China

Contributions: (I) Conception and design: Q Wang, J Wang; (II) Administrative support: Q Wang, J Wang; (III) Provision of study materials or patients: Q Wang, J Wang, J Duan, Z He; (IV) Collection and assembly of data: All authors; (V) Data analysis and interpretation: Q Wang, J Wang, C Zhang, J Duan; (VI) Manuscript writing: All authors; (VII) Final approval of manuscript: All authors.

\#These authors contributed equally to this work.

Correspondence to: Qiming Wang, MD. Department of Internal Medicine, Affiliated Cancer Hospital of Zhengzhou University, Henan Cancer Hospital, No. 127 Dongming Road, Zhengzhou 450008, China. Email: qimingwang1006@126.com; Jie Wang, MD. State Key Laboratory of Molecular Oncology, Department of Medical Oncology, National Cancer Center and Cancer Hospital, Chinese Academy of Medical Sciences and Peking Union Medical College, No. 17, Panjiayuan Nanli, Chaoyang District, Beijing 100021, China. Email: zlhuxi@163.com.

Background: Due to the high incidence and mortality of lung cancer, and etoposide is the standard firstline chemotherapy for small cell lung cancer, to evaluate the efficacy and safety of etoposide capsules at different doses as maintenance therapy for patients with extensive-stage small cell lung cancer (ES-SCLC) who show a response to etoposide plus platinum.

Methods: The study was divided into two stages: stage I, a single-center, one-arm prospective study, and stage II, a multicenter, controlled non-randomized prospective study (patients were chosen from ClinicalTrials.gov Identifier: NCT02179528). All patients received six cycles of etoposide plus platinum. Patients who were evaluated as complete remission (CR) or partial remission (PR) entered the maintenance treatment (MT) (etoposide capsule, once a day for 20 days, every 28 days as a cycle, until disease progression). In stage I, the dose of etoposide was $25 \mathrm{mg}$; in stage II, patients were non-randomized into etoposide capsule (25 mg/50 mg) and observation groups. In this study, the primary endpoints were progression-free survival (PFS) and safety; the secondary endpoint was overall survival (OS). Toxicity was graded according to the Common Terminology Criteria for Adverse Events v3.0.

Results: Ninety-two patients were enrolled. In stage I, the median PFS was 6.700 months (95\% CI: 6.408-6.992). In stage II, the median PFS of the MT group was better than that in the NMT group (8.930 vs. 5.900 months, $\mathrm{P}=0.002$ ). In the pooled analysis, the overall median PFS of the MT group was better than that of the NMT group (7.870 vs. 5.900 months, $\mathrm{P}=0.003)$. However, there was no significant difference in OS between the groups (15.030 vs. 14.330 months, $\mathrm{P}=0.813$ ). Multivariate Cox regression analysis showed that maintenance therapy was an independent protective factor for PFS in patients with ES-SCLC.

Conclusions: Etoposide capsules as maintenance therapy significantly prolonged the PFS of patients with ES-SCLC who responded to etoposide plus platinum, with acceptable tolerability.

Keywords: Etoposide capsules; extensive-stage small cell lung cancer (ES-SCLC); maintenance treatment (MT)

Submitted Dec 10, 2020. Accepted for publication Jan 21, 2021.

doi: $10.21037 /$ jtd-21-106

View this article at: http://dx.doi.org/10.21037/jtd-21-106 


\section{Introduction}

Lung cancer is the biggest contributor to cancer mortality in both men and women globally (1-4). Small cell lung cancer (SCLC) accounts for approximately $15-20 \%(5,6)$ of all lung cancer cases. It is especially sensitive to chemotherapy, with an effective rate in the range of $60-80 \%$. The majority of patients $(7,8)$ with SCLC are diagnosed with extensivestage SCLC (ES-SCLC). The biological characteristics of SCLC include poor cancer cell differentiation, rapid proliferation, a short doubling time, malignancy, a high rate of recurrence, and a short survival time (9). At diagnosis, most SCLC patients (60-70\%) have extensive-stage SCLC, which carries a median survival of approximately 10 months and a 5 -year survival rate of less than $7 \%$ (10).

The remission rate among patients who receive chemotherapy as first-line treatment for SCLC is high; however, the long-term treatment efficacy is poor (11), and almost all patients experience relapse or disease progression within 1 year after first-line treatment. Moreover, following relapse, patients are prone to multidrug resistance, and have poor sensitivity to second-line treatment. Therefore, maintenance treatment (MT) might be a suitable option for delaying disease progression and increasing the overall survival (OS) of patients with ES-SCLC who experience partial response (PR) or complete response (CR) after first-line treatment. However, many randomized trials have shown that for SCLC patients who have achieved PR and CR after first-line treatment, maintenance therapy or combination therapy has no significant survival benefit (12).

Etoposide $(3,13-16)$ is a specific antitumor drug that clinical and basic research has demonstrated to be effective in treating SCLC, malignant lymphoma, and ovarian cancer (13). Etoposide and platinum (EP) have a synergistic effect, and this combination is widely used to treat patients with SCLC (17). Studies have shown that SCLC patients with progression after intravenous treatment with an EP regimen still show benefit from oral therapy with etoposide capsules (18). Furthermore, a promising stage II clinical trial reported promising results for patients with germ cell tumors after the oral administration of etoposide capsules, with a lower-than-expected tumor recurrence rate (19).

Although immunotherapy has been demonstrated to have clinical activity in ES-SCLC, a phase 2, single-group study of maintenance pembrolizumab and a phase 3 study of ipilimumab plus chemotherapy showed no improvement in the efficacy of first-line ES-SCLC treatment (17). The standard first-line treatment for ES-SCLC consists of etoposide plus either cisplatin or carboplatin (platinumetoposide), with few alternatives (10). Thus, we conducted a prospective study to explore the benefits of etoposide capsules as maintenance therapy for patients with ES-SCLC who showed a response to first-line chemotherapy with EP.

We present the following article in accordance with the TREND reporting checklist (available at http://dx.doi. org/10.21037/jtd-21-106).

\section{Methods}

\section{Study design}

The study was divided into two stages. Stage I was a single-center, one-arm, prospective study conducted at The Affiliated Cancer Hospital of Zhengzhou University (Henan, China). Stage II was a controlled, nonrandomized, prospective phase II clinical trial involving two centers, the Department of Thoracic Medical Oncology, Peking University Cancer Hospital (Beijing, China) and The Affiliated Cancer Hospital of Zhengzhou University (Henan, China) (ClinicalTrials.gov Identifier: NCT02179528). This study was initiated by investigators. Flowcharts of stage 1 and stage 2 of the present study are shown in Figures 1 and 2, respectively. Fifteen patients were ineligible for inclusion in our study due to having a response evaluation of $\mathrm{PD}$ (progressive disease) or stable disease.

\section{Inclusion criteria}

The study inclusion criteria were as follows: (I) pathologically confirmed SCLC; (II) extensive-stage disease according to the SCLC staging system of the Veterans Association Lung Study Group (United States); (III) Eastern Cooperative Oncology Group (ECOG) performance status (PS) score of $0-2$; (IV) adequate bone marrow function, liver function, and renal function; and $(\mathrm{V})$ an estimated total survival time of $\geq 3$ months.

\section{Exclusion criteria}

The exclusion criteria were as follows: (I) pathological classification of mixed SCLC; (II) a history of malignant tumors or the presence of other tumors (not including effectively treated non-melanoma skin cancer, orthotopic cervical cancer, or malignant tumors cured by effective treatment for more than 3 years); (III) preexisting interstitial lung disease; (IV) preexisting or uncontrolled gastrointestinal 


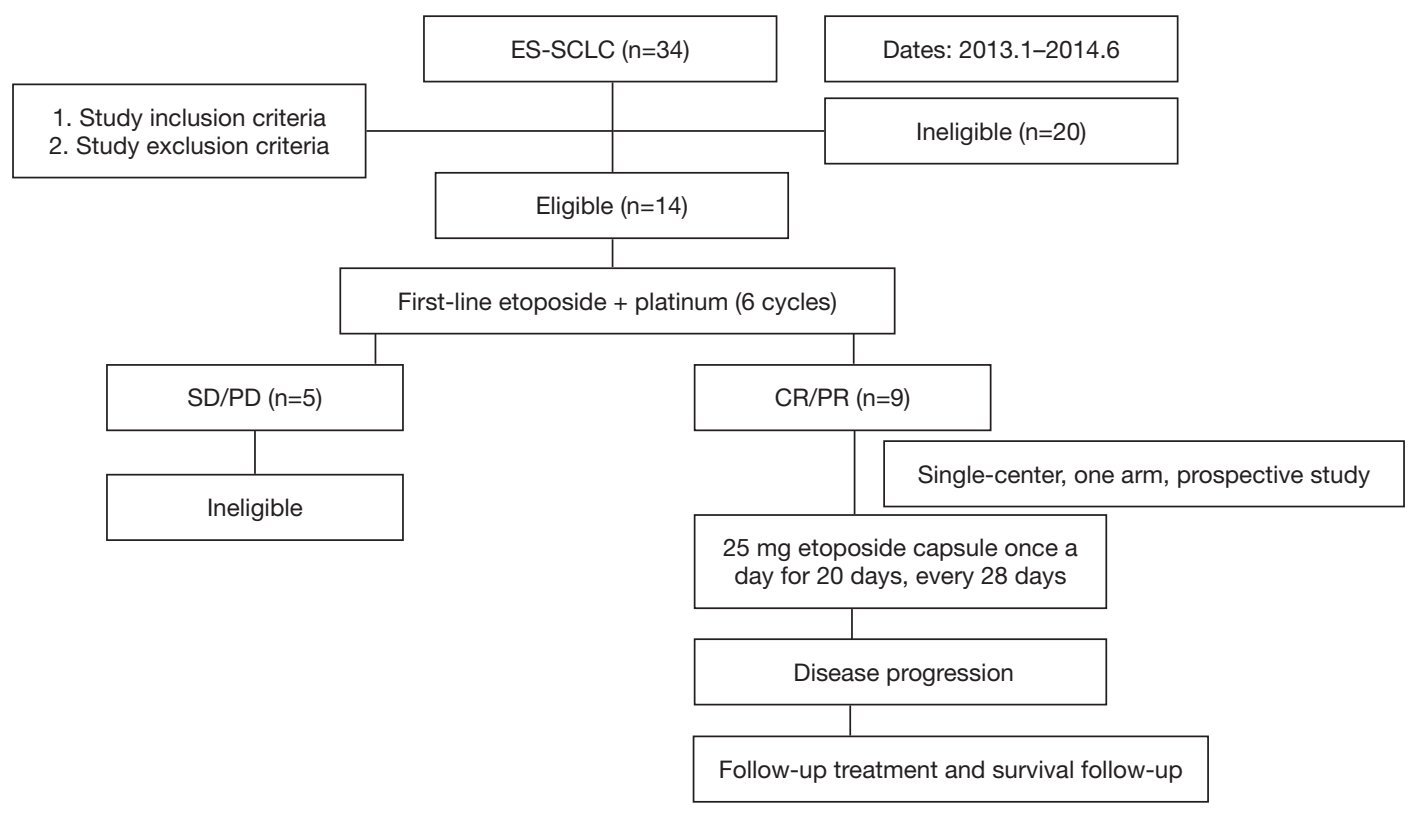

Figure 1 Study flowchart (stage I): randomized, single-center, one-arm, prospective study [The Affiliated Cancer Hospital of Zhengzhou University (Henan, China)]. ES-SCLC, extensive-stage small cell lung cancer; SD, stable disease; PD, progressive disease; CR, complete remission; PR, partial remission.

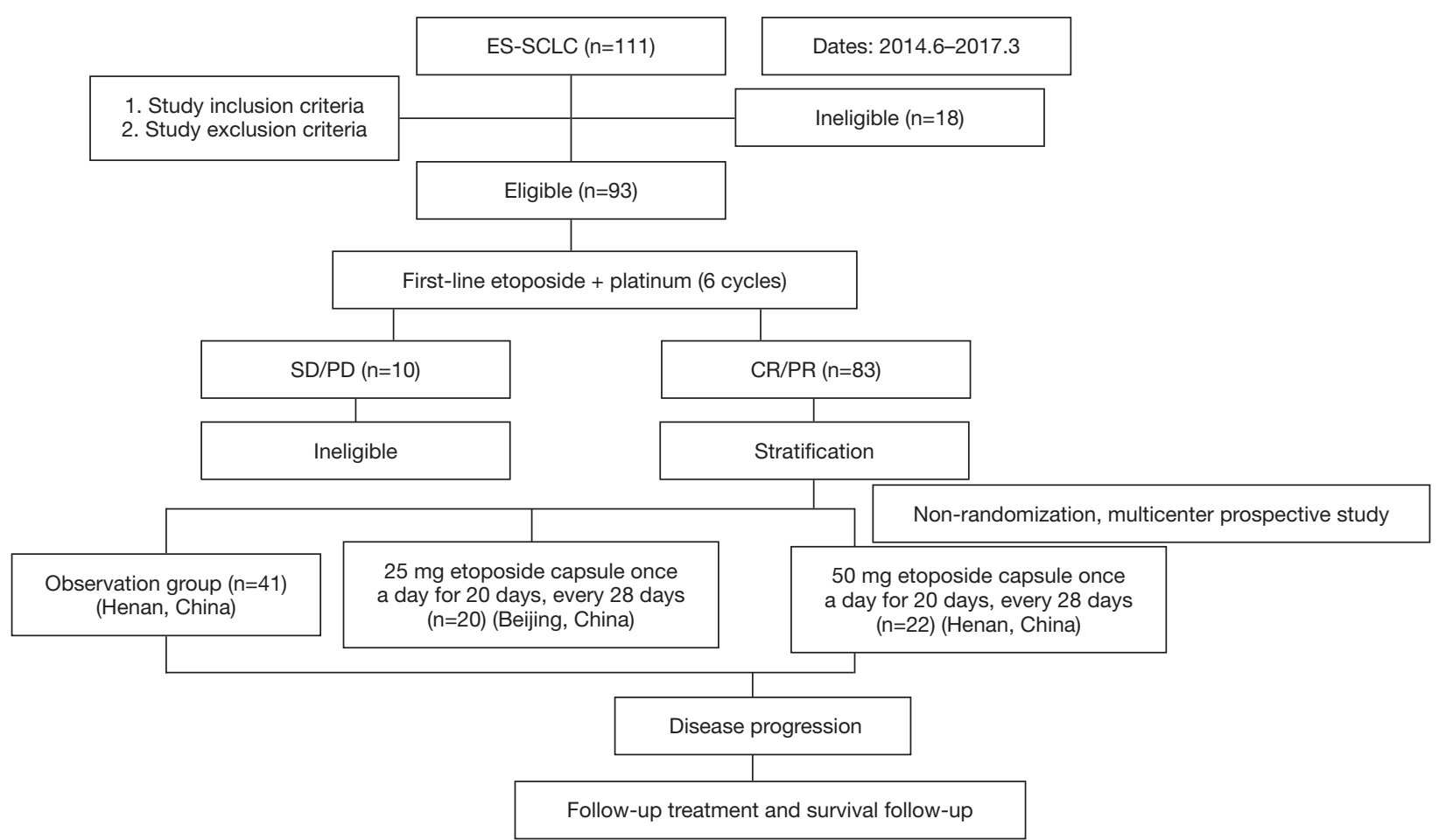

Figure 2 Study flowchart (stage II): nonrandomized, multicenter, controlled, prospective study; [1. Department of Thoracic Medical Oncology, Peking University Cancer Hospital (Beijing, China); 2. The Affiliated Cancer Hospital of Zhengzhou University (Henan, China)]. ES-SCLC, extensive-stage small cell lung cancer; SD, stable disease; PD, progressive disease; CR, complete remission; PR, partial remission. 
disorders; (V) diseases that the investigator believed would affect drug absorption; (VI) any other medical history or coexisting disease that the investigator believed could affect the patient's ability to comply with the study, or the safety or efficacy of the test drug; (VII) pregnant or lactating women; (VIII) positive results of HIV, hepatitis $\mathrm{C}$ virus, or human T-cell lymphotropic virus antibody test; (IX) positive results of hepatitis B surface antigen test; and (X) evidence of another active malignant neoplasm.

\section{Treatment and chemotherapy regimens}

Ninety-two patients in this study were included in stage I and stage II. All patients who met the inclusion criteria and the discharge criteria received six cycles of EP. Patients for whom the treatment effect was evaluated as CR or PR were included in the MT stage, with the MT being an etoposide capsule taken once a day for 20 days, every 28 days, until disease progression. In stage I, patients were given a single dose of etoposide $(25 \mathrm{mg})$. In stage II, patients were nonrandomized into the MT group (etoposide capsule (25 mg/50 mg) and the non-maintenance treatment (NMT) group (observation group). Covariates in this study included age, sex, smoking history, maintenance therapy, MT dose, and efficacy evaluation (six cycles).

\section{Endpoints}

The primary endpoints of this study were progression-free survival (PFS) and safety. The secondary study endpoint was OS, with the unit of measurement being 1 month. All eligible patients were included in the analysis of OS and PFS. The Response Evaluation Criteria in Solid Tumors (RECIST 1.1) was utilized for the evaluation of antitumor treatment efficacy (12). The evaluation of drug safety was conducted according to the National Cancer Institute (NCI) Common Terminology Criteria for Adverse Events (CTCAE), Version 4.0 (http://ctep.cancer.gov). The period from the first day of the first-line treatment until disease progression or the last follow-up was defined as PFS1. The period from the first day of etoposide capsule treatment until disease progression or the last follow-up was defined as PFS2. OS was defined as the time from the first day of the first-line treatment to the date of death from any cause.

\section{Follow-up}

The cutoff date of the last follow-up was March 15, 2019.
In the survival analysis, patients without progression or lost to follow-up at the last follow-up visit were censored from the analyses. Tumor progression or the occurrence of intolerable toxicity was recorded as an event. Efficacy evaluation were conducted every two cycles until disease progression or intolerance to toxicity occurred. The primary evaluation items included routine blood and urine tests, liver and kidney function tests, electrocardiogram, hepatobiliary and pancreatic cervical lymphadenopathy, whole-body bone scan, head computed tomography (CT), and magnetic resonance imaging (MRI) examination.

\section{Ethics and informed consent}

This study was initiated by investigators. The investigations were approved by the ethics committees of the Affiliated Cancer Hospital of Zhengzhou University, and Peking University Cancer Hospital. Signed written informed consent was obtained from all patients before participation in the study. This study was conducted in accordance with the ethical principles of the Declaration of Helsinki (as revised in 2013) and the International Conference on Harmonization of Good Clinical Practice guidelines.

\section{Statistical analysis}

Statistical analysis of all randomly assigned eligible patients was performed. Survival curves were estimated using the Kaplan-Meier method; a log-rank test was used for comparison and analysis; and a chi-square test was used to evaluate adverse events. Subsequently, a Cox proportional hazards model was used to calculate the PFS of the two treatment groups. The results were expressed as hazard ratios (HRs) and 95\% confidence intervals (CIs). All data analyses were performed with SPSS version 22.0 (IBM SPSS Statistics for Windows, version 22.0, released in 2013; IBM Corp., Armonk, NY, USA). A P value of less than 0.05 was considered to represent a statistically significant difference. All statistical $\mathrm{P}$ values were two-sided.

\section{Results}

\section{Patients}

Our study was conducted from January 1, 2013, to March 31, 2017, at the Affiliated Cancer Hospital of Zhengzhou University and the Department of Medical Oncology, Peking University Cancer Hospital. The median follow-up 
Table 1 Baseline demographic and clinical characteristics of patients in the stage I and stage II studies

\begin{tabular}{|c|c|c|c|c|}
\hline Characteristic & Stage I, n (\%) & \multicolumn{3}{|c|}{ Stage II } \\
\hline Age (years) & & & & 0.743 \\
\hline$<60$ & $5(55.56)$ & $20(24.1)$ & $21(25.3)$ & \\
\hline$\geq 60$ & $4(44.44)$ & $22(26.5)$ & $20(24.1)$ & \\
\hline Male & $5(55.56)$ & $24(28.9)$ & $29(34.9)$ & \\
\hline Female & $4(44.44)$ & $18(21.7)$ & $12(14.5)$ & \\
\hline Smoking history & & & & 0.218 \\
\hline Former light smoker/other smoker & $5(55.56)$ & $21(25.3)$ & $26(31.3)$ & \\
\hline PR & $9(100.00)$ & $42(50.60)$ & $39(47.00)$ & \\
\hline CR & $0(0.00)$ & $0(0.00)$ & $2(2.40)$ & \\
\hline
\end{tabular}

MT, maintenance treatment; NMT, non-maintenance treatment; EP, etoposide and platinum; PR, partial remission; CR, complete remission.

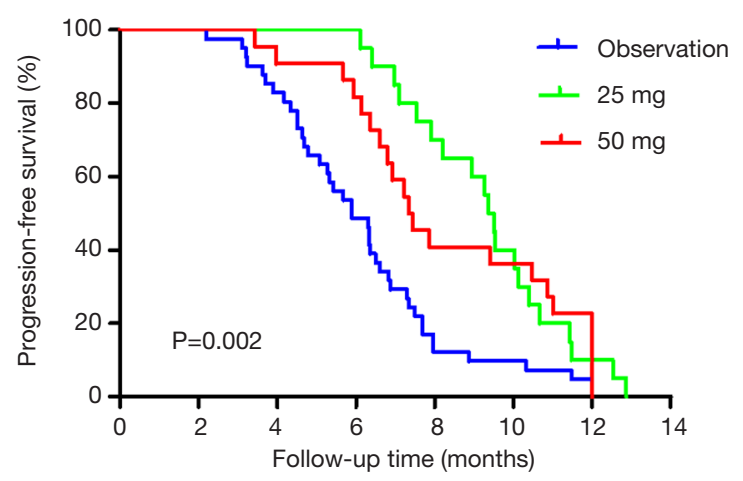

Figure 3 Stage II: multicenter, nonrandomized, controlled trial (50/25 mg etoposide capsule and observation group). The median PFS was better in the maintenance group than in the nonmaintenance group (8.930 vs. 5.900 months, log-rank test $\mathrm{P}=0.002$, HR: 2.272, 95\% CI: 1.445-3.572). No difference was observed in the median PFS between 25 and $50 \mathrm{mg}$ (9.435 vs. 7.380 months, $\log$-rank test $\mathrm{P}>0.05)$. PFS, progression-free survival.

time was 28 months, and the cutoff date of the last followup was March 15, 2019.

We screened 145 patients with ES-SCLC 92 patients were finally enrolled in our study. Fifty-three patients who were ineligible were excluded. Reasons for ineligibility included: age under 18 years $(\mathrm{n}=6)$; stage IA $(\mathrm{n}=16)$, stage IB $(\mathrm{n}=8)$, or stage IIIB $(\mathrm{n}=8)$ disease; and evaluated as SD $(\mathrm{n}=9)$ or PD ( $\mathrm{n}=6)$ after 6 cycles of the EP regimen (Figures 1,2).

Of the 92 patients included in the final analysis, $9(9.78 \%)$ were from stage I and 83 (90.22\%) were from stage II. The demographic characteristics of the patients involved in stage I (Table 1) and stage II (Table 1) were evenly distributed.

\section{Efficacy}

In stage I, the median PFS was 6.700 months (HR: 0.146, 95\% CI: 6.408-6.992), and the median OS was 15.030 months (HR: 0.089, 95\% CI: 14.855-15.205).

In stage II, the median PFS in the MT group was longer than that in the NMT group (8.930 vs. 5.900 months, logrank test $\mathrm{P}=0.002$ ) (Figure 3). The overall median PFS was 7.100 months (HR: 2.272, 95\% CI: $1.445-3.572$ ). OS showed no significant difference between the two groups (15.270 vs. 14.330 months, log-rank test $\mathrm{P}=0.695$ ). The overall median OS was 14.970 months (HR: 0.792, 95\% CI: 13.515-16.425).

For the pooled analysis of stages I and II, the median PFS in the MT group was longer than that in the NMT group (the observation group) (7.870 vs. 5.900 months, log-rank test $\mathrm{P}=0.003$ ) (Figure 4). The overall median PFS 


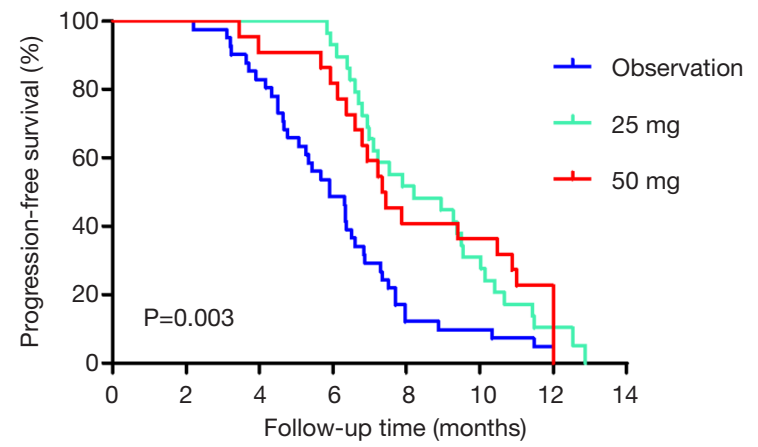

Figure 4 In the pooled analysis of stages I and II, the median PFS in the maintenance treatment group $(25 \mathrm{mg}$ group $+50 \mathrm{mg}$ group) was better than that in the non-maintenance treatment group (observation group) (7.870 vs. 5.900 months, log-rank test $\mathrm{P}=0.003)$. No difference was observed in the median PFS between 25 and $50 \mathrm{mg}$ (8.200 vs. 7.380 months, log-rank test $\mathrm{P}>0.05$, HR: 1.450, 95\% CI: 0.633-3.322). PFS, progression-free survival.

was 6.930 months (HR: 1.450, 95\% CI: 0.633-3.322). No significant difference was found in OS between the two groups (15.030 vs. 14.330 months, log-rank test $\mathrm{P}=0.813$ ) (Figure 5). The overall median OS was 15.030 months (HR: 1.054, 95\% CI: 0.679-1.637).

There was no significant difference in PFS2 (2.330 vs. 2.530 months, log-rank test $\mathrm{P}=0.211$ ) between the $25 \mathrm{mg}$ group and the $50 \mathrm{mg}$ group. The overall median PFS2 was 2.400 months (HR: 0.194, 95\% CI: 2.020-2.780).

Univariate analysis showed that age, sex, smoking history, maintenance therapy status, and best efficacy evaluation had no effect on PFS. Maintenance therapy had a positive effect on the PFS of all patients $(\mathrm{P}<0.001)$, although no similar effect was observed for OS $(\mathrm{P}=0.813)$.

For all patients (Table 2) and for stage II patients (Table 3), multivariate Cox regression analysis show that age, sex, smoking history, or evaluation efficacy results during the treatment of EP had no effect on PFS, and none of these factors were an independent predictor of PFS. However, maintenance therapy status was an independent predictor of PFS in all patients and in stage II patients. Maintenance therapy had a negative regression coefficient, which indicated it to be an independent protective factor for PFS in patients with ES-SCLC $(\mathrm{P}<0.001)$.

\section{Safety}

The main adverse events recorded in this study were

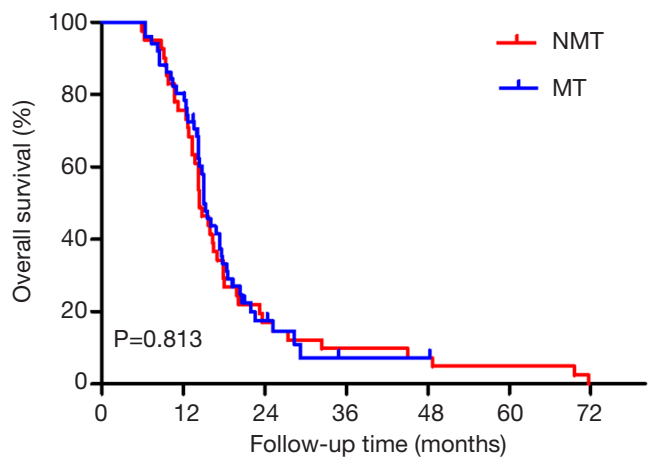

Figure 5 In the pooled analysis of stages I and II, there was no significant difference in OS between the groups (15.030 vs. 14.330 months, log-rank test $\mathrm{P}=0.813$, HR: 1.054 , 95\% CI: 0.679 1.637). MT, maintenance treatment; NMT, non-maintenance treatment; OS, overall survival.

myelosuppression, liver and kidney function damage, and gastrointestinal tract symptoms. Most adverse events were grade I-II, and the incidence of grade III-IV adverse events was low. All adverse reactions were tolerable, with no serious adverse events leading to death. There was no significant difference in the incidence of decreased leukocyte count, decreased neutrophil count, decreased platelet count, decreased hemoglobin count, digestive tract events, aspartate aminotransferase elevation, or alanine aminotransferase enzyme elevation between the NMT and MT groups (all P>0.05, Table 4). All adverse reactions were relieved after symptomatic treatment without drug interruption.

\section{Discussion}

Our study reached the primary endpoints successfully. In the pooled analysis, the median PFS of the MT group reached 7.870 months, showing that the EP regimen should be considered as the preferred MT option for patients with ES-SCLC. At present, no standard protocol for maintenance therapy of SCLC exists, and a number of randomized trials have demonstrated that maintenance therapy and combination therapy have no survival benefit for ES-SCLC patients (14). In our study, stage I, a prospective, single-center clinical study, showed that the median PFS and median OS were 6.700 and 15.030 months, respectively, which were superior to those reported by other 
Table 2 Multivariate Cox regression analysis of stage I and stage II

\begin{tabular}{|c|c|c|c|c|}
\hline Characteristic & \multicolumn{2}{|l|}{ PFS } & \multicolumn{2}{|l|}{ OS } \\
\hline Sex & $1.279(0.610-2.681)$ & 0.515 & $0.958(0.440-2.083)$ & 0.913 \\
\hline Smoking history & $1.079(0.528-2.204)$ & 0.836 & $1.119(0.525-2.384)$ & 0.771 \\
\hline Response to EP & $1.599(0.352-7.264)$ & 0.543 & $0.518(0.111-2.405)$ & 0.401 \\
\hline
\end{tabular}

PFS, progression-free survival; OS, overall survival; EP, etoposide and platinum.

Table 3 Multivariate Cox regression analysis of stage II

\begin{tabular}{lcccc}
\hline \multirow{2}{*}{ Characteristic } & \multicolumn{2}{c}{ PFS } & \multicolumn{2}{c}{ OS } \\
\cline { 2 - 3 } & $\mathrm{HR}(95 \% \mathrm{Cl})$ & $\mathrm{P}$ & $\mathrm{HR}(95 \% \mathrm{Cl})$ & $\mathrm{P}$ \\
\hline Sex & $1.214(0.560-2.628)$ & 0.623 & $0.980(0.443-2.166)$ & 0.960 \\
Smoking history & $1.066(0.508-2.240)$ & 0.866 & $1.085(0.502-2.347)$ & 0.836 \\
Response to EP & $1.603(0.353-7.285)$ & 0.541 & $0.508(0.109-2.371)$ & 0.389 \\
Maintenance therapy (yes or no) & $0.384(0.232-0.634)$ & $<0.001$ & $0.898(0.551-1.464)$ & 0.665 \\
\hline
\end{tabular}

PFS, progression-free survival; OS, overall survival; EP, etoposide and platinum.

Table 4 Grade 3-4 adverse reactions in the MT and NMT groups (stage I and stage II)

\begin{tabular}{lcccc}
\hline Adverse events & MT & NMT & \\
& $(\mathrm{n}=51)$ & $(\mathrm{n}=41)$ & $\mathrm{P}$ \\
\hline Decreased leukocytes & 2 & 1 & 0.691 \\
Decreased absolute neutrophil numbers & 3 & 2 & 0.833 \\
Decreased platelets & 4 & 5 & 0.485 \\
Decreased hemoglobin & 6 & 2 & 0.244 \\
Constipation & 3 & 1 & 0.421 \\
Increased aspartate aminotransferase & 2 & 1 & 0.691 \\
Alanine aminotransferase enzyme elevation & 4 & 1 & 0.258 \\
\hline MT, maintenance treatment; NMT, non-maintenance treatment.
\end{tabular}

clinical studies (14). Our prospective study demonstrated significantly improved PFS; therefore, we reached the primary endpoint. In our stage II study, the median PFS of the MT and NMT groups was 8.930 and 5.900 months, respectively, which represented a significant improvement from that reported in previous studies. Therefore, our multicenter, exploratory study demonstrated improved PFS in patients who received maintenance therapy. The pooled analysis of stage I and II showed that maintenance therapy could significantly prolong PFS, which is inconsistent with the findings of a study (8) by Gadgeel et al. Although our study did not reach its secondary endpoint, it did reach the primary endpoint.

In our study, the median PFS2 was 2.400 months. Our results were similar to Qi et al.'s study (20); however, our study differs from other studies as it included only patients with extensive disease and consisted of two stages, both of which were prospective studies. Further exploration with the inclusion of the survival time of patients with ES-SCLC is needed.

With a median retention time of 2.4 months, patients in the MT group in our study received 6 treatment cycles of EP, which was significantly lower than the median of 11 cycles and 12 cycles of treatment in Dómine and Turgeon's study $(21,22)$. Therefore, we can surmise that the survival benefit may not be closely related to the number of EP treatment cycles. Univariate analysis in our study showed that there was a statistically significant improvement of PFS in maintenance therapy; multivariate analysis showed that maintenance therapy was an independent predictor of PFS. In brief, the results of our study's univariate and multivariate analyses indicated that maintenance therapy was better than non-maintenance therapy in terms of PFS benefit, whereas age, sex, smoking history, MT dose, and evaluation efficacy 
results during the use of EP had no association with PFS.

In our study, we gave an etoposide capsule $(25 \mathrm{mg})$ once daily as the maintenance therapy for ES-SCLC patients who experienced CR or PR after 6 cycles of EP. With a bioavailability of $50 \%$ (23), oral etoposide capsules have the same efficacy and toxicity as the same dose of etoposide administered intravenously. Compared with the group administered single high-dose etoposide, the etoposide administration group showed a better remission rate, remission duration, and 5-day survival (days $1-5$ ), and the overall remission rate was $89 \%(\mathrm{P}<0.001)$. Therefore, this study clearly demonstrated the importance of the etoposide dosing regimen (24).

Etoposide is a cell-cycle-specific drug. As shown in another study, etoposide exhibits time-dependent antitumor activity, so prolonging its duration of action can increase the antitumor effects (25). At a high concentration (i.e., $10 \mathrm{mg} / \mathrm{mL}$ or higher), our pharmacokinetic goal was to maintain etoposide concentrations between $0.5-1.0 \mathrm{mg} / \mathrm{mL}$ in the plasma for several hours. Etoposide capsules $50 \mathrm{mg}$ (24) appear to be a suitable dose choice, which was inconsistent with the results of our study. Our study found no significant difference in OS between patients administered etoposide capsules of the two different doses. Although there was no increased toxic side effect induced by the higher dose, it did not bring about OS benefit and failed to prolong survival. Moreover, increasing the maintenance dose may increase the financial burden on the patient and reduce their compliance. Therefore, for ES-SCLC patients who experience CR or PR after six cycles of EP, we recommend giving an etoposide capsule $25 \mathrm{mg}$ once daily until disease progression or the occurrence of an intolerable adverse event.

In our study, myelosuppression, impairment of liver and kidney function, and gastrointestinal symptoms (nausea and vomiting) were common adverse reactions to etoposide capsule therapy. No serious adverse events of grade IIIIV occurred, and all adverse events were tolerable after symptomatic treatment.

Our study has the following shortcomings that need to be discussed: (I) the sample size was small; (II) the stage I study lacked a control group; (III) the stage I study was a singlecenter study, while the stage II study was a prospective, nonrandomized, exploratory study with an offset; (IV) a sample size estimate was not calculated; and (V) there is no exploration of the possible mechanism of this therapy. The advantages of our study included the following: (I) our investigations were conducted by researchers; (II) all research was prospective; and (III) stage I was a singlecenter, prospective study, and stage II was a multicenter, prospective, randomized study.

In conclusion, etoposide capsules as maintenance therapy could significantly prolong the PFS of patients with ESSCLC who showed a response to EP, and this treatment had acceptable tolerability. We reached the primary study endpoints but did not reach the secondary endpoint, which may be because OS is influenced by many factors, including follow-up treatment after disease progression. Longer PFS is likely to extend OS, so more research needs to be carried out in the future. Furthermore, etoposide capsules have already been on the market for years, and the prescription can be easily obtained. We recommend giving an etoposide capsule $25 \mathrm{mg}$ once daily until the patient experiences disease progression or an intolerable adverse event.

\section{Acknowledgments}

Funding: This work was supported in part by the Henan Thousand Talents Program (2019), the National Natural Science Foundation of China (No. 81272600), the National Basic Research Program of China (No. 2012CB933304), Henan Provincial Training Abroad Foundation for Leaders of Medical Science (No. 201082), Henan Provincial Special Funds for Health and Technological Innovative Talents (No. 2011020155), Henan Provincial Research Program of Application Foundation and Advanced Technology (No. 112300410033), a project co-sponsored by the Henan Province and Ministry of Health of Medical Science and Technology Program (No. 201601026), the 51282 project Leading Talent of Henan Provincial Health Science and Technology Innovation Talents (No. [2016]32), and Jieping Wu Medical Foundation for Clinical Research (No. 320.6799.15018). We also received support from the Program for Science and Technology Innovation Talents in Universities of Henan Province (No. 18HASTIT044). The funders had no role in the study design, data collection and analysis, decision to publish, or preparation of the manuscript.

\section{Footnote}

Reporting Checklist: The authors have completed the TREND reporting checklist. Available at http://dx.doi. org/10.21037/jtd-21-106

Data Sharing Statement: Available at http://dx.doi. 
org/10.21037/jtd-21-106

Conflicts of Interest: All authors have completed the ICMJE uniform disclosure form (available at http://dx.doi. org/10.21037/jtd-21-106). The authors have no conflicts of interest to declare.

Ethical Statement: The authors are accountable for all aspects of the work in ensuring that questions related to the accuracy or integrity of any part of the work are appropriately investigated and resolved. The investigations were approved by the ethics committees of the Affiliated Cancer Hospital of Zhengzhou University, and Peking University Cancer Hospital. Signed written informed consent was obtained from all patients before participation in the study. This study was conducted in accordance with the ethical principles of the Declaration of Helsinki (as revised in 2013) and the International Conference on Harmonization of Good Clinical Practice guidelines.

Open Access Statement: This is an Open Access article distributed in accordance with the Creative Commons Attribution-NonCommercial-NoDerivs 4.0 International License (CC BY-NC-ND 4.0), which permits the noncommercial replication and distribution of the article with the strict proviso that no changes or edits are made and the original work is properly cited (including links to both the formal publication through the relevant DOI and the license). See: https://creativecommons.org/licenses/by-nc-nd/4.0/.

\section{References}

1. Blair HA. Atezolizumab: A review in previously treated advanced non-small cell lung cancer. Target Oncol 2018;13:399-407.

2. Kawaguchi T, Mack PC. Ethnic difference in lung cancer: an important issue in a globalized society. J Thorac Dis 2020;12:3774-5.

3. Jin J, Si J, Liu Y, et al. Elevated serum soluble programmed cell death ligand 1 concentration as a potential marker for poor prognosis in small cell lung cancer patients with chemotherapy. Respir Res 2018;19:197.

4. Pacheco J, Bunn PA. Advancements in small cell lung cancer: the changing landscape following IMPOWER-133. Clinical Lung Cancer 2019;20:148-160.e2.

5. Luo H, Zhang L, Yang B, et al. A randomized phase 2 trial of apatinib vs observation as maintenance treatment following first-line induction chemotherapy in extensive-stage small cell lung cancer. Invest New Drugs 2020;38:148-59.

6. Tiseo M, Boni L, Ambrosio F, et al. Italian, multicenter, phase III, randomized study of cisplatin plus etoposide with or without bevacizumab as first-line treatment in extensive-stage small-cell lung cancer: The GOIRC-AIFA FARM6PMFJM Trial. J Clin Oncol 2017;35:1281-7.

7. Thomas M, Ponce-Aix S, Navarro A, et al. Immunotherapeutic maintenance treatment with toll-like receptor 9 agonist lefitolimod in patients with extensivestage small-cell lung cancer: results from the exploratory, controlled, randomized, international phase II IMPULSE study. Ann Oncol 2018;29:2076-84.

8. Gadgeel SM, Pennell NA, Fidler MJ, et al. Phase II study of maintenance pembrolizumab in patients with extensivestage small cell lung cancer (SCLC). J Thorac Oncol 2018;13:1393-9.

9. Sen T, Della Corte CM, Milutinovic S, et al. Combination treatment of the oral CHK1 inhibitor, SRA737 and low dose gemcitabine, enhances the effect of PD-L1 blockade by modulating the immune microenvironment in small cell lung cancer. J Thorac Oncol 2019;14:2152-63.

10. Paz-Ares L, Dvorkin M, Chen Y, et al. Durvalumab plus platinum-etoposide versus platinum-etoposide in firstline treatment of extensive-stage small-cell lung cancer (CASPIAN): a randomized, controlled, open-label, phase 3 trial. Lancet 2019;394:1929-39.

11. Armstrong SA, Liu SV. Immune checkpoint inhibitors in small cell lung cancer: a partially realized potential. Adv Ther 2019;36:1826-32.

12. Houdek $\breve{S}$, Buchler T, Kindlova E. Comparison of RECIST 1.1 and iRECIST for response evaluation in solid tumors. Klin Onkol 2017;30:32-9.

13. Li D, Liu L. Bioequivalence study of two formulations of etoposide in advanced lung cancer patients. Int J Clin Pharmacol Ther 2015;53:474-9.

14. Higgins KA, Gorgens S, Sudmeier LJ, et al. Recent developments in limited stage small cell lung cancer. Transl Lung Cancer Res 2019;8:S147-52.

15. Cai Y, Wan L, Yang J, et al. High-dose etoposide could discriminate the benefit from autologous peripheral blood stem cell transplantation in the patients with refractory diffuse large B cell lymphoma. Ann Hematol 2019;98:823-31.

16. Nemade H, Chaudhari U, Acharya A, et al. Cell death mechanisms of the anti-cancer drug etoposide on human cardiomyocytes isolated from pluripotent stem cells. Arch Toxicol 2018;92:1507-24. 
17. Horn L, Mansfield AS, Szczęsna A, et al. First-Line atezolizumab plus chemotherapy in extensive-stage smallcell lung cancer. N Engl J Med 2018;379:2220-9.

18. Honecker F, Aparicio J, Berney D, et al. ESMO Consensus Conference on testicular germ cell cancer: diagnosis, treatment and follow-up. Ann Oncol 2018;29:1658-86.

19. Kalra M, Adra N, Hanna N, et al. High-dose chemotherapy plus peripheral blood stem cell transplantation for patients with relapsed germ cell tumors and active brain metastases. Cancer 2020;126:1202-7.

20. Qi J, Xu L, Sun J, et al. Thoracic radiotherapy benefits elderly extensive-stage small cell lung cancer patients with distant metastasis. Cancer Manag Res 2019;11:10767-75.

21. Dómine M, Moran T, Isla D, et al. SEOM clinical guidelines for the treatment of small-cell lung cancer (SCLC) (2019). Clin Transl Oncol 2020;22:245-55.

22. Turgeon GA, Souhami L, Kopek N, et al. Thoracic irradiation in 3weeks for limited-stage small cell lung

Cite this article as: Zhang C, Duan J, He Z, Yang L, Yang S, Zhang Z, Liu Y, Wan R, Lin L, Wu X, Wang W, Wang Q, Wang J. The benefits of etoposide capsules as maintenance therapy for patients with extensive-stage small cell lung cancer: a prospective two-stage, two-center study. J Thorac Dis 2021;13(1):343-352. doi: 10.21037/jtd-21-106 cancer: Is twice a day fractionation really needed? Cancer Radiother 2017;21:89-98.

23. Jain AS, Dhawan VV, Sarmento B, et al. In vitro and ex vivo evaluations of lipid anti-cancer nanoformulations: insights and assessment of bioavailability enhancement. AAPS PharmSciTech 2016;17:553-71.

24. Li L, Li Q, Xu Y, et al. Phase II study of oral etoposide maintenance for patients with extensive stage small cell lung cancer who have responded to the induction on an EP regimen. Thoracic Cancer 2013;4:234-40.

25. Rudin CM, Awad MM, Navarro A, et al. Pembrolizumab or placebo plus etoposide and platinum as first-line therapy for extensive-stage small-cell lung cancer: randomized, double-blind, phase III KEYNOTE-604 study. J Clin Oncol 2020;38:2369-79.

(English Language Editor: J. Reynolds) 